\title{
A Similarity-Driven Transfer System
}

\author{
Hideo Watarlabe \\ IBM Research, Tokyo Research Isaboratory \\ 5-19 Sanbancho, Chiyoda-ku, Tokyo 102 Japan \\ e-mail: watanabe@trl.vnet.ibm.com
}

\begin{abstract}
The transfer phase in machine translation (MT) systems has been considered to be more complicated than analysis and generation, since it is inherently a. conglomeration of individual lexical rules. Currently some attempts are being made to use case-based reasoning in machine translation, that is, to make decisions on the basis of translation examples at appro priate points in MT. This paper proposes a new type of transfer system, called a Similarity-driven Transfer System (SimTran), for use in such case-based MT' (CBMT).
\end{abstract}

\section{Introduction}

The transfer process in machine translation systems is, in general, more complicated than the processes of analysis and generation. One reason for this is that it relies heavily on human heuristic knowledge or the linguistic intuition of the rule writers. Unfortunately, linguistic intuition tends to be unable to control the process properly for a wide variety of inputs, because of the huge amount of data and the huge number of situations that need to be considered. However, rule writers must rely on their linguistic intuition to some extent, because there is no linguistic theory on lexical transfer [7]. Another reason [8][13] is that the transfer task is inherently a congiomeration of individual lexical rules. Therefore, the transfer process can be said to fall into a class of problem that cannot easily be controlled by the linguistic intuition of rule writers.

In accordance with these observations, various attempts have been made to overcome the problems of transfer; they include knowledge-based MT [12], bilingual signs [13], and Tags for MT[1]. One such approach is case-based or example-based MT [4] [9] [10] [11]. The essential idea behind all case-based MT (CBM'T) methods is that the system chooses the case (or example) most similar to the given in put from the case base, and applies the knowledge attached to the chosen case to the in put. ${ }^{1}$

Supposing that there is a corpus of parsed translation examples in which corresponding parts are linked to each other, we can regard those parsed transla-

\footnotetext{
1 This approach can be regarded as an application of casebased reasoning [3] to natural language tranglation.
}

tion examples as translation rules. A promising approach is therefore to make a transfer process that (1) chooses a set of translation examples, each source part of which is similar to a part of the input, and all source parts of which overlap the whole input, and (2) constructs an output by combining the target parts of those translation examples chosen. However, this does not mean that existing transfer knowledge. should be abandoned. Rather, such transfer knowledge should be used as a fail-safe mechanism if there are no appropriate examples. In the similarity-driven transfer system (Simtran) we have developed, both translation examples and existing transfer knowledge are treated uniformly as translation patterns, and are called translation rules.

In Figure 1, for example, (a) is the parsed dependency structure of an input Japanese sentence, "kare ga kusuri wo nomu." Suppose that (b) is selected as the most similar transiation rule for the part "kare ga ... nomu" from the translation rule-base, and that (c) is selected as the most similar translation rule for the part "kusuri wo nomu," even though there are several translation candidates for the Japanese verb "nomu." This figure illustrates what we would like to do; that is, to construct (d), the transiated structure by combining the target structures of the selected translation rules.

fo develop this kind of system, we must consider the following issues:

(a) a metric for similarity,

(b) a mechanism for combining target parts of rules, and

(c) correspondence between the source part and the target part of a rule.

'To handle the last two issues, I developed a model called Rules Combination Thansfer (RCT) [14]. Sim Tran is RCT coupled with a similarity calculation method. In this paper, I will introduce RCT and the similarity calculation method used in Sim Trun.

The next section defines the data structure for graphs, and the format of a translation rule. Section 3 presents a method for calculating the similarity between an input and the source part of a translation rule. Section 4 describes the flow of the transfer process in RCT. Section 5 gives examples of translation using SimTran, and Section 6 discusses related work. Some concluding remarks bring the paper to an end. 


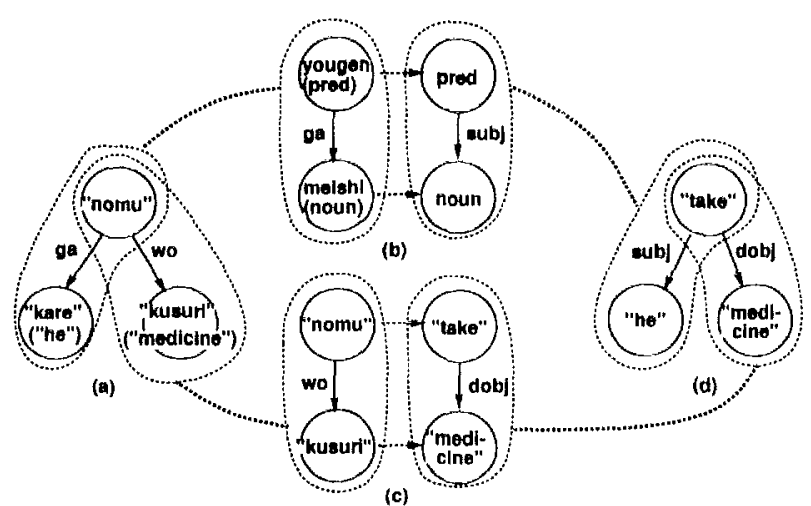

Figure 1: Sample Japanest-to. Jinglish transtation

\section{Translation Rules}

$A$ basic type of graph used in this paper is a labeled directed graph, or an ldg. ${ }^{2}$ An ldg G consists of a set of nodes $N$, and a set of $\operatorname{arcs} A$. Further, each node and are has a label. In particular, node labels are unique. Fach node consists of features, each of which is a pair of a feature name and a feature value.

If an Idg has only one root node, then it is called an rldg, and if an ldg has no cyclic path, then it is called an Idag. 'I'herefore, an rldag denotes an ldg that has only one root node and no cyclic path.

A translation rule ${ }^{4} \mathrm{r}$ consists of the following three components:

$$
r=\left\langle G_{r m}, M, G_{c}\right\rangle
$$

where $G_{m}$ is a matching graph, $G_{c}$ is a coustruction graph, and $M$ is a set of mappings between $G_{m}$ and Gi:

$\Lambda$ matching graph $G_{m}$ and a construction graph $C_{i}$ must be at least an rldag. ${ }^{5}$ Further, nodes in $G_{m}$ must be labeled miquely; that is, each node in $G_{m}$ must have only one urique label, and the label of the node $n_{c}$ in $G_{c}$ is determined to be the label of the

\footnotetext{
'The term 'labeled' tneaus that nodes and arcs are laticled, and the term 'directed' meany that each arc has a direction. Further, an ldy in this puper refers to a connected graph unless otlerwise specified.

STlie term dag is often used in the NLP world, and usually denotes a rooted connected labeled (as functional) directed graph. But in this paper, dag denotes a directed acyclic grapli that may have multiple roots, is not necessarily a connected gruph, and does not iecessarily have labels.

In this paper, the term rule does not menn $n$ procedure, but rather a pattern of translation knowledge.

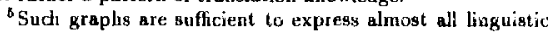
structures.
}

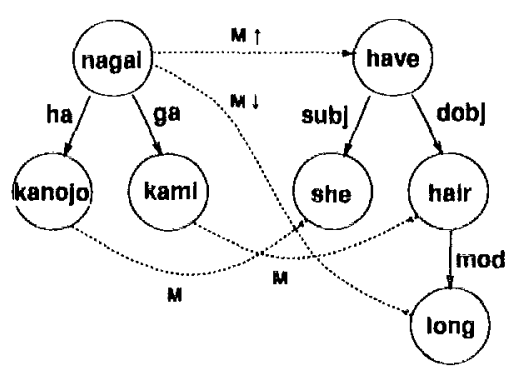

Figure 2: Sample rule for translation betwesen Japanese and English

mode $n_{m}$ in $C_{m}$ such that $n_{r}=M\left(n_{m}\right)$.

Mapping between $G_{m}$ and $G_{c}$ designates the cor. pespondences between undes in $C_{m}$ and $C_{r}$. For instance, in Figure 2, the Japanese word "nagai" ("long") should correspond to both of the Finglish words "heve" and "long," because if another word governs the word "nagai" then its Knglish translat tion should be comnected to the word "have." On the other hand, if the Japanese word "totemo" ("very") modifies "naga!" then its linglish transhation "very" should be connected to "long." This shows that for a node in a source language, two kinds of connection point, for translations of both governing structures and governed structures of the node, are needed in its translated structure. 'I his implies that there should be two kinds of corres] pondence between $G_{m}$ and $G_{C 1}$ namely, (1) a mapping from a $G_{n \downarrow}$ node $n_{m}$ to a $G_{c}$ node $n_{c}$ that is to be a node connected to translations 
of structures governing $n_{m}$, and (2) a mapping from $n_{m}$ to a $G_{c}$ node $n_{c}^{\prime}$ that is to be a node connected to translations of structures governed by $n_{m}$. We call the former an upward mapping and the latter a downward mapping, and denote these two kinds of mapping as follows:

$$
M=\langle M \uparrow, M \downarrow\rangle
$$

where $M \uparrow$ is upward mapping, and $M \downarrow$ is downward mapping.

Not all kinds of mapping should be permitted as $M \uparrow$ and $M \downarrow$. A translation rule $r=\left\langle G_{m}, M_{1} G_{c}\right\rangle$ must satisfy the following conditions:

(1) $M \uparrow$ and $M \downarrow$ are both injections,

(2) there are no two distinct nodes $x$ and $y$ in $G_{m}$ such that $\mathbf{M}(\mathbf{x})=\mathbf{M}(\mathbf{y}),{ }^{B}$ and

(3) $M \uparrow\left(\operatorname{root}\left(G_{m b}\right)\right)=\operatorname{root}\left(G_{c}^{4}\right)$.

Condition (1) ensures that there is only one connection point in $G_{o}$ for each translation of governing structures and governed structures, condition (2) ensures that the label of a $G_{c}$ rode is determined uniquely, and condition (3) ensures that the result of this transfer model becomes a rooted graph (sec [15] for details). A rule satisying these conditions is said to be sōund.

\section{Similarity Calculation}

This section desribes how a similarity is calculated.

\subsection{Graph Distance}

The similarity between a $G_{m}$ and an input graph $G_{i r}$ is defined as the inverse of the graph distance ${ }^{7}$ between thern. First, the simple graph distance $D_{g}^{\prime}$ hetween $G_{i n}$ and $G_{m}$ is given as follows:

$$
\begin{aligned}
& D_{g}^{\prime}\left(G_{i n}, G_{m}\right)=D_{n}\left(R_{i n}, R_{m}\right) \\
& \quad+\sum_{a_{m}} \min \left(D_{g}^{\prime}\left(G S\left(K_{i n}, a_{m}\right), G S\left(R_{m}, a_{m}\right)\right)\right)
\end{aligned}
$$

where $R_{i n}$ and $R_{m}$ are roots of $G_{i n}$ and $G_{m}$, respectively, $D_{n}$ is a node distance, $a_{m}$ is an arc in $G_{m}$ such that its source node is $R_{m}$, and $G S(n, a)$ denotes a subgraph that is related to an arc $a$ from $n$.

Briefly, a simple distance is the sum of the node distance between two roots and the sum of the minimal simple distances between $G_{i n}$ subgraphs and $G_{m}$ subgraphs that, for each arc $a$ outgoing from the $G_{m}$ root node, are related to the all arcs $a$ from the root nodes.

\footnotetext{
This means that either $M \uparrow(x)$ or $M L(x)$ is equal to either $M \uparrow(y)$ or $M \downarrow(y)$

${ }^{7}$ Distances defined in this section ure not actual distances in the mathematical sense.
}

However, the larger $G_{m}$ is, the larger this simple distance becomes. 'Therefore, when normalized by the number of nodes in $G_{m}$, the graph distance $D_{g}$ is given as follows:

$$
D_{g}\left(G_{i n}, G_{m}\right)=\frac{D_{g}^{\prime}\left(G_{i n}, G_{m}\right)}{N}
$$

where $N$ is the number of nodes in $G_{m}$.

\subsection{Node Distance}

When considering the distance between two words (nodes), we usually think of their semantic distance in a semantic hicrarchy. In general, no matter what semantic hierarchy we use, it is inevitable that there will be some sort of distortion. Further, as stated be fore, a node consists of several features and may not have a lexical form that is a pointer to a semantic hierarchy. 'Therefore, a promising approach to calculating distances between nodes is to use both a semantic hierarchy and syntactic features, that is, to use syntactic features to correct the distortion contained in the semantic hierarchy to some extent.

The node distance between a $G_{i n}$ node $n_{i}$ and a $G_{n}$ node $n_{m}$ is defined as follows:

$$
D_{n}\left(n_{i}, n_{m}\right)=\frac{D_{f}+D_{s} * \delta_{s}}{N_{f}+\delta_{s}}
$$

where $D_{f}$ is a feature node distance, $D_{s}$ is a semantic node distance, $N_{f}$ is the number of features in $n_{m}$ for $D_{f}$, and $\delta_{s}$ is the weight of a semantic distance.

'The semantic distance $D_{s}$ between a $G_{i n}$ word $w_{i n}$ and a $G_{m}$ word $w_{m}$ is given by the following equation. In Sim Tran, Buntui Goi Hyou [5] code (or bghcode ${ }^{8}$ ) is used for calculating the semantic distance between Japanese words.

$$
\begin{aligned}
& D_{o}\left(w_{i n}, w_{m}\right)= \\
& \begin{cases}0 & w_{i n}=w_{m} \\
0.5 & w_{i n} \text { or } w_{m} \text { is unknown } \\
1 & w_{i n} \text { and } w_{m} \text { are unknown } \\
\frac{\left|\operatorname{lgh}\left(w_{i n}\right)-b g h\left(w_{s}\right)\right|+\delta_{b}}{b g h m a x+\delta_{h}} & \text { otherwise. }\end{cases}
\end{aligned}
$$

where $b g h(w)$ is the fraction part of the bghcode of $w$, bghmax is the maximal difference between two bghcode fraction parts, and $\delta_{b}$ is a penalty incurred if two words are not identical.

The feature distance $D_{f}$ between a $G_{i n}$ node $n_{i n}$ and a $C_{m}$ norle $n_{m}$ is given as follows:

$$
\begin{aligned}
& D_{f}\left(n_{i n}, n_{m}\right)=\sum_{f \in n_{m}} d_{f}\left(n_{i n}, f\right) \\
& d_{f}\left(n_{i n}, f n: f v\right)= \\
& \begin{cases}1 \quad f_{i n}\left(f n_{i n}: f v_{i n}\right) \text { whose } f n_{i n}=f n, \text { and } \\
\text { fvis consistent with } f v_{i n} \\
0 \quad \text { otherwise }\end{cases}
\end{aligned}
$$

\footnotetext{
${ }^{8} \mathrm{~A}$ bghcode is a fraction of number. Its integer part roughly corresponds to a ayntactic category, and therefore, only its fraction part is used.
} 


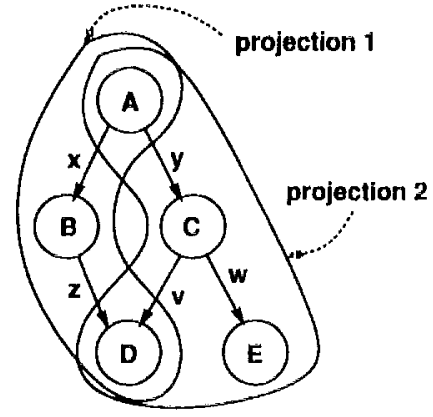

Figure 3: An isomorphic cover

In the above equation, the consistency checking de pends on a feature.

\section{Rules Combination Transfer}

In this section, I present the flow of the transduction process by using RCT formalism.

\subsection{Rule Selection}

A transfer process must first find a sot of rules whosi $G_{m}$ s' matching parts (called projections) totilly overlap an input structure, and which is the most similar to the input. We call a union of projections a cover, and a cover identical to the input an isomorphic cover (or i-cover). In orther words, what we want here is the $i$-cover that is the most similar to the imput. Further, if a $C_{m}$ make a projection $p j$ on a $G_{i n}$, then the $G_{m}$ is called the origin graph of the $p j$. A pivot is a node of $G_{i n}$ that has more than one origin graph, and a matching pivot is the: origin node of a pivot. For instance, in Figure $3, \Lambda$ and $\mathrm{D}$ are pivots.

There may be some methods for finding such an $\mathrm{i}$. cover rule set. One method is to pick up a rule whose projection does not have any arc overlapped by a cover by other selected rules until there are no incovered arcs, if it is desirable that a rule set should have few overlaps as possible. Wo have also developed another method using dynamic programming, which can choose the most similar rule set from candidate rule sets. Briefly, it stores the most similar rule set for each combination of arcs of each node from leaves up to the root, and the most similar rule set stored in the root node is the one for the input structure (see [6] for details).
Fach matching pivot in a similar i-cover rule set must have $M$ or $M \downarrow$, to ensure that the $G_{C} s$ of the cover rute set produce a connected graph as a result. If there are rules in the given i-cover rule set that do not satisfy this condition, they are removed from the set of rulo candidates, and the cover search method is executed until an i-cover rule sot that satisfies this condition is found. Such an i-cover rule set is called a proper rule set.

Next, for each projection of the given i-cover, we must natake a copy of its origin rule, or rule instance, be cause one rule maty maks more than one projection on $C_{i \text { in }}$.

\subsection{Pre-Lexicalization}

It may liapen that a lexieal-form of a $C_{c}$ in the given rule instance is not it candidate translation word of its correspondiug word in the input, because a lexical form in a matchimg node in its $G_{m}$ is not necessarily the same as the input word. In this case, such a node is lexicalized by candidate translation words.

\subsection{Node Labeling}

i'he label of a $a_{m}$ node becomes the label of its matching mote in $C_{i+1}$. Since $C_{i n}$ norles are labeled uniçuely, $C_{m}$ nodes are also labeled uniquely. On the other hand, the label of a $C_{c}$ node $\pi_{c}$ becomes the $t_{\mathrm{t}-}$ bel of a $i_{n t}$ joode $\left(n_{m}\right)$ such that $n_{n}=M \uparrow\left(n_{m}\right)$ or $n_{r:}=: M\left\lfloor\left(n_{m}\right)\right.$.

T'here may, however, be two nodes in $C_{c}$ in a rult instance that are mapped by a node in $G_{m}$ with $M$ and $\boldsymbol{M}$ t, respectively. In the succeeding process, $\boldsymbol{G}_{c}$ nodes with the same label are merged into one node in order to generate an output structure. In this phase, the transferred labels of these two nodes should be different, becalse the two nodes should not be merged for this rule. Wo must therefore relabel $G_{c}$ nodes of rule instances as follows:

$G_{r}$ Node Relabeling: for any label $l$ in $G_{c}$, if $l$ is distributed to two distinct nodes of $\left(r_{r}\right.$ by both $M$ । and $M \mid$ from it rode of $G_{m}$, then a labed $l$ in it $G_{C}$ node, which is mapped only by $M T$, or is mapped by both $M+$ and $M \perp$ and has no descendisnts, is changed to $l^{\prime}$.

\section{d. G Gluing}

Unification is a well-known computational tool for connecting graphs, and is widely used in natural language processing. Usually, mification uses two func 


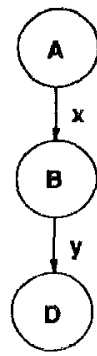

(a)

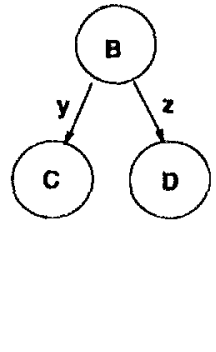

(b)

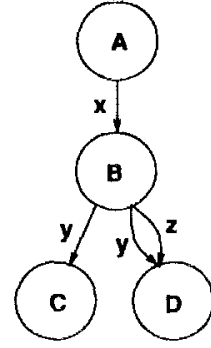

(c) Gluing of (a) and (b)
Figure 4: Example of gluing

tional rldags as data and unifies them from the root node down to the leaves. In RCT, however, we want to merge those nodes of two graphs that have the same labels, even if their root nodes are different, and they are not functional, as shown in Figure 4. Unification, however, cannot proceed in this manner, be cause it unifies two nodes that occupy the same position, and always starts from the root node. For instance, in Figure 4, even if unification starts from node $B$ then it fails, since it tries to unify node $D$ of (a) and node $\mathrm{C}$ of (b) for arc $\mathrm{y}$.

In Graph Grammars, this method of connecting two graphs is called gluing [2]. The ghuing used in Graph Grammars is not concerned with the content of a node, so it must be extended in order to check the consistency among the nodes to be glued. In SimTran, if two features conflict then the feature whose rule is more similar to the input is taken.

Briefly, gluing is performed as follows ${ }^{9}$ : Firsi, nodes with the same label are merged if they are consistent. If any nodes fail to be merged, then the gluing also fails. If all the merges succeed, all ares are reattached to the original nodes, which may or may not be meryed. As a result, some arcs with the same labels and attached to the same nodes may be merged, if they are consistent.

A glued graph is not necessarily a connected, rooted, or acyclic graph, but we usually need a connected rldag in natural language processing. Several constraints satisfying such requirements are described in previous papers [14][15].

After the $G_{f} 3$ have been labeled and relabeled, the target structure is built by gluing the $G_{c} \mathrm{~s}$.

\footnotetext{
Details of the algorithm are given it previous papers [14][15].
}

\subsection{Post-Lexicalization}

The constructed target structure is still imperfect; there might be a $G_{c}$ node that has no lexical-form, because there are some rules made from transfer knowledge that have no lexical-forms. Therefore, as in the pre-lexicalization phase, non-lexical $G_{0}$ nodes are lexicalized.

\section{Examples}

This section gives examples of translation by Sim Tran. Figure 5 shows how the Japanese sentence "Kanojo no me ga totemo kireina no wo sitteirı" is translated into the English senterce "(I) know that she has very beautiful eyes." In this figure, (a) is an input sentence structure, (b), (c), and (d) are rules (precisely, rule instances), and (e) is the output structure produced. In these rules, a mapping line not marked $M \uparrow$ and $M \downarrow$ has both $M \uparrow$ and $M \downarrow$. Dotted lines designate matching or gluing correspondences between rule nodes and input or output norles, respectively. Further, numbers prefixed by "*' denote node labels. In this example, we assume type hierarchies in which, for instance, 'yougen(predicate)' is a super-category of 'keiyou(adj)', and "kanojo(she)" is an instance of 'human'. Note that the node labels of both "have" in rule instance (c) and lower 'pred' in rule instance (b) are changed from that of the corresponding Japanese word "kirei(beautiful)" by the $G_{c}$ node relabeling procedure.

Another example is shown in Figure 6 , which shows how the Japanese sentence "LS ga ... wo fusegu tame ni buhin ni kanzei wo kakeru" is translated into the Englisit sentence "US imposes tax on parts in order to blockade ... ." In this example, (a) is an input structure, (b), (c) and (d) are matched rules, and (e) is the output structure prodiced. 'The Japanese verb "kakeru" has several translation candidates associated with different governing words, as shown in the following table:

\begin{tabular}{|l|c|}
\hline Similarity & Japanese-English \\
\hline \hline 5.988 & $\begin{array}{c}\text { (meishi) ni zeikin wo kakeru } \\
\text { impose tax on (noun) }\end{array}$ \\
\hline 3.077 & $\begin{array}{c}\text { (meishi) wo saiban ni kakeru } \\
\text { take (noun) to court }\end{array}$ \\
\hline 2.717 & $\begin{array}{c}\text { (meishi) wo mado ni kakeru } \\
\text { hang (noun) in window }\end{array}$ \\
\hline 2.545 & $\begin{array}{c}\text { (meishi) wo sutoobu ni kakeru } \\
\text { put (noun) on stove }\end{array}$ \\
\hline 2.040 & $\begin{array}{c}\text { hankati ni kousui wo kakeru } \\
\text { spray perfume on hand kerchief }\end{array}$ \\
\hline
\end{tabular}

'This table lists the top five similar rules for the part "buhin ni kanzei wo kakeru" of the input. As shown 


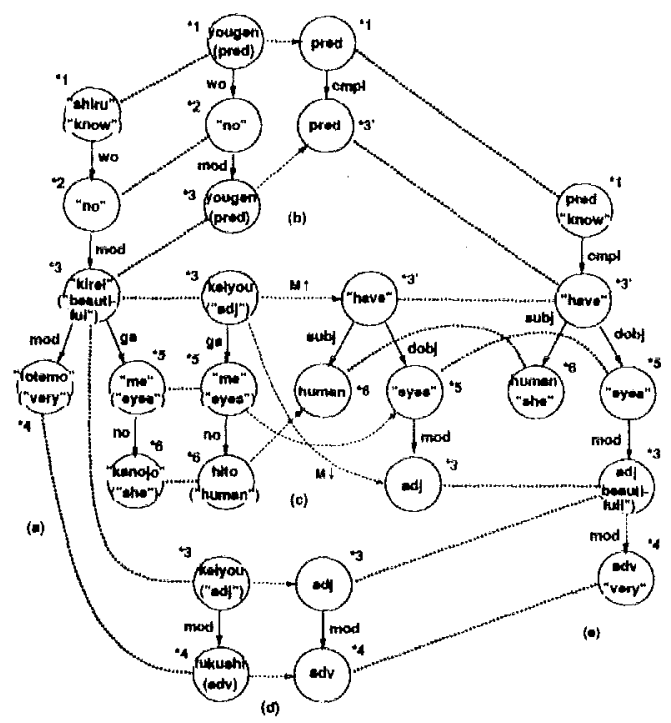

Figure 5: Fixample 1 of transtation by Sim Tiven

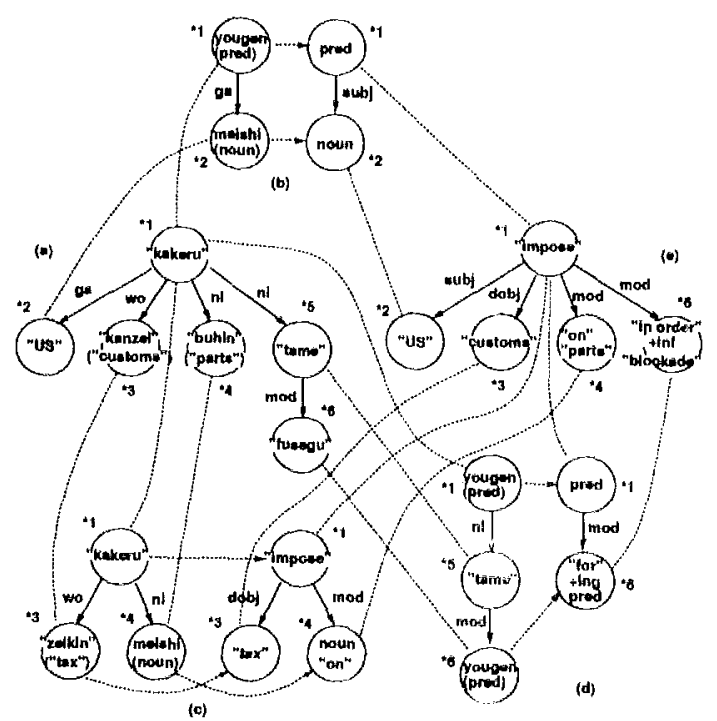

Figure 6: Example 2 of trans]ation by Sim Tiun 
in this table, rule (c) is the most similar one. Note that this similarity calculation was done for all rules, including non-lexical translation rules. There were no appropriate example rules for the part "US ga kakeru," and a non-lexical rule (b) was therefore selected. Further, note that the lexical forms in $* 3$ nodes of (c) and (e) are different, and that *d node of (c) has no lexical form other than a preposition, whereas ${ }^{*} 4$ node of (e) has a lexical form. 'The former was obtained by pre-lexicalization, and the latter by post-lexicalization.

\section{Related Work}

Although there were several early experimental projects on CBMT [4][9][11], MBT-II [10] is the first working prototype of a case-based transfer system, and demonstrates the promise of the CBMT approach. It uses Japanege-to-English translation examples as translation rules, chooses the source trees of examples that are most similar to the input tree from the root node down to the leaves, and assembles those target, trees to produce an output tree. With respect to the transducing mechanism, MB'T-II is a tree-totree transducer adopting one-to-one correspondence.

MT by LTAGs [1], although it is not an sttempt of CRMT', proposed a similar mechanism to RCT described in this paper. It uses paired derivation trees of English and French as translation rules. An input sentence is parsed by the source grammar, and at the same time, its output tree is generated by derivation pairs of trees used in the parsing. As a transducer, this mechanism is also a tree-to-tree transducer adopting one-to-one correspondence.

In contrast, the RCT employed in Sim Trun is a rldagto-rldag transducer adopting upward and downward correspondences. These extended correspondences are desirable for expressing the structural discrepancies that often occur in translation. Moreover, this transducing model is a parallel production system [2] that can produce an output structure in one execution of gluing if all the $G_{c}$ s required to produce an output are supplied.

\section{Conclusion}

In this paper, 1 described a case-based transfer system, Sim Iran, which combines RCT with a similarity calculation method. RCT has powerful correspondences between the source structure and the target structure of a translation rule, which can express most structural discrepancies between two languages. As a transducing mechanism, RCT is a parallel nondestructive rldag-to-rldag transducing system. I also propose a similarity calculation method for graphs whose nodes consist of syntactic and semantic features, and show that a translation rule that has no lexical forms can bo used as a default rule, that is, that such rules can provide a fail-safe mechanism if there are no appropriate translation examples.

\section{References}

[1] Abeille, A., Schabes, Y., and Joshi, A. K., "Using lexicalized Tags for Machine Translation," Proc. of Coling 90, 1990.

[2] Ehrig, H., "Introduction to the Algebraic Theory of Graph Grammars," Proc of International Workshop on Grapì Grammars, INCS 73, 1-69, 1979.

[d] Kolodner, J. and Riesbeck, C., "Case-Based Reasoning," tutorial textbook of $11 \mathrm{th}$ IJCAI, 1089.

[4] Nagao, M., "A Framework of a Mechanical Transiation between Jupanese and English by Analogy Principle," Elithorn, A. and Banerji, R. (eds.): Artificial and Human Intelligence, NATO 1984.

[5] National Language Research Institute: Bunrui Goi Hyou (in Japanese), Syuuei Syuppan, 1964.

[6] Maruyama, H. and Watanabe, H., "Tree Cover Search Algorithm for Example-Based Transintion," Proc. of th Int. Conf. on Theoretical and Methodological Isstes in Machine Translation, 1992.

[7] Melby, A K., "Iexical Transfer: A Missing Element in Linguistic Theories," Proc. of Coling 86 , pp. 104$106,1986$.

[8] Nitta, Y., "Idiosyncratic Gap: A 'Tough Problem to Structure-bound Machine Translation," P'roc. of $\mathrm{Col}$ ing $86, \mathrm{pp} .107-111,1986$.

[9] Sadler, V., "Working with Analogical Semantics," Foris Publications, 1989

[10] Sato, S. and Nagao, M., "Foward Memory-based Translation," Coling 90, 1990.

[11] Sumita, E., Iida, H, and Kohyama, II., "Translating with Examples: A New Approach to Machine Translation," Prac. of Info Japan 90, 1990.

[12] Tomita, M. and Carbonell, J. G., "Another Stride lowards Knowledge-Based Machine Iranslation," Proc. of Coling 86,1986

[13] Tsujii, J, and Fujita, K., "Lexical 'Iransfer based on bilingual signs: Towards interaction during transfer," Proc. of Seoul Int. Conf, on NLP, 1990.

[14] Watanabe, II., "A Model of a Transfer Y'rocess Using Combinations of Translation Rules," Proc of Pacific Rim of Int. Conf. on AI' 90,1990

[15] Watanabe, H., "A Formal Model of Transfer Using Rules Combination," submitted to Machine Translation, 1991. 